\title{
Extended middle pancreatectomy for a large pancreatic cystic neoplasm: a case report
}

Albert KK Chui ${ }^{*}$, FRACS, MD, Juanita N Chui ${ }^{2}$, BSC (Adv), MD, Gregory E Antonio ${ }^{3}$, FHKAM (Radiology), MD, $\mathrm{KC} \mathrm{Lam}{ }^{4}, \mathrm{MB}, \mathrm{BS}$, FHKAM (Surgery)

\author{
${ }^{1}$ Private Practice \\ ${ }^{2}$ School of Medicine, University of Sydney, Sydney, Australia \\ ${ }^{3}$ Department of Radiology, St Teresa's Hospital, Hong Kong \\ ${ }^{4}$ Private Practice
}

Hong Kong Med J 2020;26:535-7

https://doi.org/10.12809/hkmj208372

\section{Case report}

In December 2016, a 47-year-old Chinese woman was referred to our clinic for treatment of a large cystic pancreatic lesion. She presented with a 12-month history of intermittent epigastric pain associated with eating and weight loss of $5 \mathrm{~kg}$. The patient had a history of uterine fibroids and underwent myomectomy 10 years previously. She was also under observation for a benign thyroid nodule. She had no family history of malignancy, denied consumption of alcohol, was a non-smoker, and lived as a housewife.

A contrast-enhanced computed tomography scan of the abdomen revealed a cystic neoplasm $8 \mathrm{~cm}$ in diameter involving the head, neck, and body of the pancreas, with proximal dilatation of the pancreatic duct (Fig 1). Based on the patient's symptoms and the size of the lesion, surgical resection was recommended. Preoperative laboratory test results,

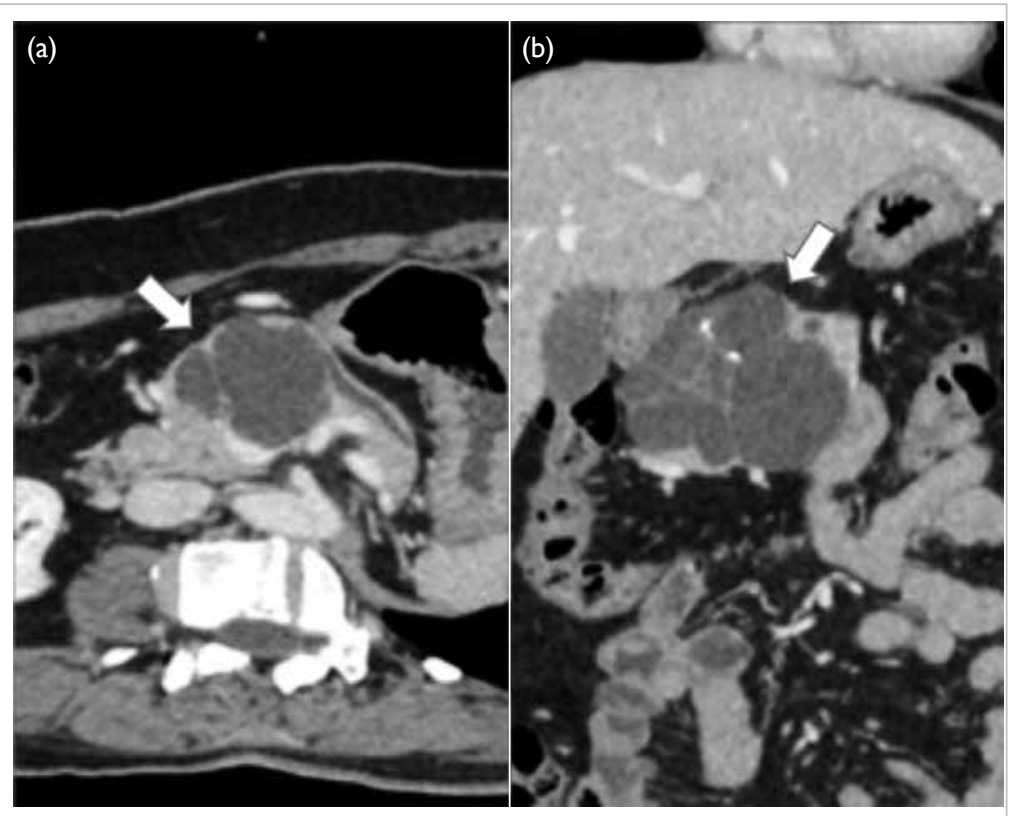

FIG I. Multiplanar reformatted oblique computed tomography (a) axial and (b) coronal images showing a multilocular complex cystic lesion (arrows) centred in the pancreatic neck. The pancreatic duct proximal to this mass was dilated including full blood count, liver and renal function tests, blood glucose level, amylase, carcinoembryonic antigen, and cancer antigen 19.9, were within normal limits.

During surgery, a multiloculated cystic lesion was identified (Fig 2). The cystic content was serous. No nearby enlarged lymph nodes or tissue invasion suggestive of frank malignancy were evident. The pancreatic tail was healthy. An extended middle pancreatectomy (EMP) was performed. The patient recovered without complication and was discharged from hospital 13 days after surgery. Histological examination of the resected tissue confirmed a benign serous cystadenoma.

\section{Surgical procedure}

A bilateral subcostal incision was made. The lesser sac was entered to expose the anterior aspect of the pancreas after division of the gastrocolic ligament (Fig 2a). The gastroduodenal junction was purposefully divided to gain better exposure of the large cystic lesion and to facilitate further pancreatic dissection (Fig $2 \mathrm{~b}$ ). The posterior peritoneum along the inferior and superior margins of the pancreas was dissected. The superior mesenteric vein was identified under the neck of the pancreas. The splenic vein was carefully divided away from the gland and all the small branches of the pancreas draining into the splenic vein were ligated. The gallbladder was removed to facilitate identification and mobilisation of the common bile duct, the hepatic artery, and the portal vein from above. The involved portion of the pancreas was mobilised on both cephalic and caudal sides. The cystic tumour was resected in its entirety with a margin by scalpel and cautery. Most of the head (estimated 80\%-90\%), neck, and body of the pancreas were removed (Fig 2c). The remaining pancreatic head stump was carefully over-sewn with 3-O Prolene sutures (Ethicon; Cornelia [GA], United States) to avoid pancreatic leak. The pancreatic duct opening was identified and separately sutured. The stump was further re-enforced with fibrin sealant, Tisseel glue (Baxter Healthcare, Deerfield 

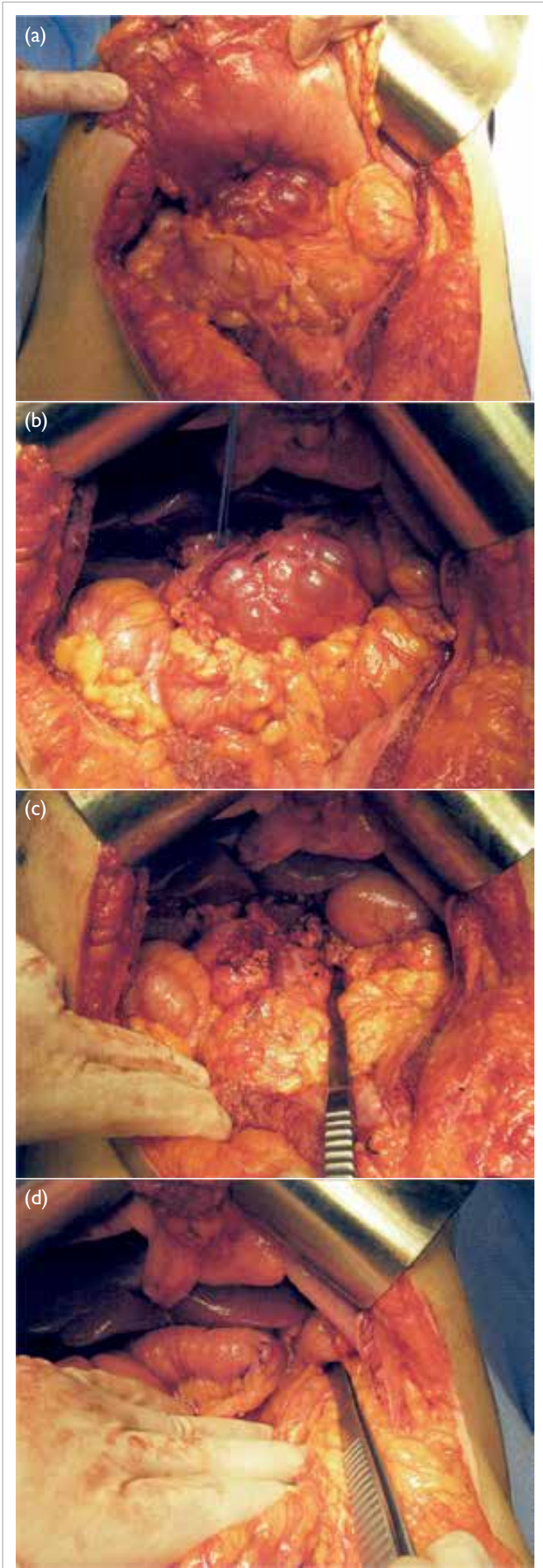

FIG 2. Intraoperative photographs. (a) The large pancreatic cystic neoplasm evident within the lesser sac. (b) The gastroduodenal junction was divided. (c) The cystic tumour has been resected. The portal vein and stumps of pancreas on either end are exposed. (d) Pancreatojejunostomy with the stump of the distal pancreas invaginated inside the jejunum
[IL], United States). The distal side stump was anastomosed into a Roux loop of jejunum using an end-to-end technique with invagination (Fig 2d). A short segment of catheter was inserted into the pancreatic duct. The gastric pylorus was then joined up to the proximal jejunum as a gastrojejunostomy and a jejunojejunostomy was fashioned to prevent bile reflux. The proximal jejunum was reconnected to the jejunal Roux loop distally. Two drainage tubes were placed close to the closed cephalic stump and the pancreaticojejunostomy anastomosis before proceeding with abdominal wound closure.

\section{Discussion}

For benign or low-grade malignant lesions of the pancreatic neck and body, traditional approaches for surgical resection include a pancreaticoduodenectomy (PD) or an extended distal pancreatectomy (EDP). Enucleation is suitable only for small and superficial lesions that do not have any connection with the pancreatic duct. In 1957, Guillemin and Bessot first reported the technique of middle pancreatectomy (MP) for chronic pancreatitis and pancreatic transection injury. ${ }^{1}$ In 1982, Dagradi and Serio proposed the use of MP for resection of benign tumours or tumours with low malignant potential situated in the pancreatic neck and body. ${ }^{2}$ The technique has since gained acceptance.

In this case, the large cystadenoma involved a significant portion of the head of the pancreas in addition to the neck and the body. Traditional approaches for resection of such a lesion would involve either subtotal pancreatectomy or PD. The MP technique described in the literature has not included resection of the head of the pancreas. However, in this case, most of the pancreatic head in addition to the neck and the body of pancreas had to be resected. Therefore, the procedure is being formally named for the first time, EMP.

Extended middle pancreatectomy permits resection of a lesion that extends into the head of the pancreas while conferring similar advantages of an MP over traditional approaches. Compared with other surgical options (subtotal pancreatectomy and PD), this confers the advantage of parenchymal preservation and consequent preservation of pancreatic exocrine and endocrine functions. ${ }^{3}$ Pancreaticoduodenectomy has been associated with increased postoperative morbidity and mortality. Similarly, the inability to preserve the spleen in subtotal pancreatectomy has been associated with complications of thrombosis and susceptibility to infection. Finally, MP has been associated with a higher incidence of pancreatic fistula, compared with PD and extended distal pancreatectomy procedures. ${ }^{4,5}$ This has been attributed to the need to manage two pancreatic remnants by anastomosis or closure. However, as much less pancreatic head 
tissue is left behind in EMP, the risk of pancreatic leak from the proximal stump should theoretically be lower than that observed in MP.

We report, to the best of our knowledge, the first clinical case of EMP in the management of a large cystic neoplasm. This case demonstrated excellent postoperative outcomes and suggests that EMP may be considered a viable preferred surgical option in selected cases. However, the technical difficulties of this procedure should not be underestimated. The long-term functional outcomes have yet to be substantiated by further clinical experience.

\section{Author contributions}

All authors contributed to the concept or design of the study, acquisition of the data, analysis or interpretation of the data, drafting of the manuscript, and critical revision of the manuscript for important content. All authors had full access to the data, contributed to the study, approved the final version for publication, and take responsibility for its accuracy and integrity.

\section{Conflicts of interest}

As an editor of the journal, AKK Chui was not involved in the peer review process. The other authors have disclosed no conflicts of interest.

\section{Funding/support}

This case report received no specific grant from any funding agency in the public, commercial, or not-for-profit sectors.

\section{Ethics approval}

The patient was treated in accordance with the tenets of the Declaration of Helsinki. Patient consent was obtained.

\section{References}

1. Guillemin P, Bessot M. Chronic calcifying pancreatitis in renal tuberculosis: pancreatojejunostomy using an original technic [in French]. Mem Acad Chir (Paris) 1957;83:86971.

2. Dagradi A, Serio G. Pancreatectomia intermedia. In: Enciclopedia Medica Italiana. Pancreas, vol XI. Florence: USES Ed Scientifiche; 1984: 850-1.

3. Tan Z, Chen P, Dong Z, Zhou B, Guo WD. Clinical efficacy of middle pancreatectomy contrasts distal pancreatectomy: a single-institution experience and review of literature. ANZ J Surg 2019;89:E184-9.

4. Shibata S, Sato T, Andoh H, et al. Outcomes and indications of segmental pancreatectomy. Comparison with distal pancreatectomy. Dig Surg 2004;21:48-53.

5. Du ZY, Chen S, Han BS, Shen BY, Liu YB, Peng CH. Middle segmental pancreatectomy: a safe and organ-preserving option foe benign and low-grade malignant lesions. World J Gastroenterol 2013;19:1458-65. 